\title{
Consumption habits of pregnant women and implications for developmental biology: a survey of predominantly Hispanic women in California
}

Sarah E Santiago, Grace H Park and Kelly J Huffman*

\begin{abstract}
Background: Healthy post-pregnancy outcomes are contingent upon an informed regimen of prenatal care encouraging healthy maternal consumption habits. In this article, we describe aspects of maternal intake of food, drink, and medication in a population of predominantly Hispanic women in Southern California. Potential implications for unhealthy prenatal dietary choices are discussed.

Methods: The Food, Beverage, and Medication Intake Questionnaire (FBMIQ) measures common practices of maternal consumption during pregnancy. The FBMIQ was administered to English and Spanish speaking pregnant and recently pregnant ( 36 weeks pregnant - 8 weeks post-partum) women over the age of 18 who were receiving care from a private medical group in Downey CA.

Results: A total of 200 women completed the FBMIQ. Consumption habits of healthy foods and beverages, unhealthy foods, unhealthy beverages, and medication are characterized in this article. Data indicate widespread consumption of fresh fruit, meats, milk and juice and indicate most women used prenatal vitamin supplements. Studies in developmental neuroscience have shown that certain substances may cause teratogenic effects on the fetus when ingested by the mother during pregnancy. Those potentially harmful substances included in our study were Bisphenol-A (BPA), methylmercury, caffeine, alcohol and certain medications. Our results show that a proportion of the women surveyed in our study consumed BPA, methylmercury, caffeine, alcohol, and certain medications at varied levels during pregnancy. This represents an interesting finding and suggests a disconnect between scientific data and general recommendations provided to pregnant mothers by obstetricians.

Conclusions: The results of our study demonstrate that a proportion of pregnant women consume substances that are potentially teratogenic and may impact the health and well being of the offspring. It is important to appraise healthy and unhealthy consumption habits in order to encourage healthy practices and alleviate future effects of preventable, toxin-induced developmental issues. Prenatal advising should discourage the consumption of dangerous foods, beverages, and medications that women commonly report eating during pregnancy.
\end{abstract}

Keywords: Prenatal exposure, Maternal diet, Environmental toxins, Fetal health

\footnotetext{
* Correspondence: kelly.huffman@ucr.edu

Department of Psychology, University of California, 900 University Avenue,

Riverside 92521-0128, CA, California
}

\section{Ciomed Central




\section{Background}

Post-partum outcomes for mother and child are linked to maternal consumption habits during pregnancy $[1,2]$. Thus, it is imperative that pregnant women be informed of the risks and benefits of certain dietary practices. This is not a simple task, as many foods, beverages and medications carry unknown risks [3]. Additionally, most primary care physicians and obstetricians are not aware of the dietary and over-the-counter medication intake practices of their patients and thus lack the information needed to help guide them. Assessment of common practices of food, drink, and medication intake during pregnancy informs the direction of preventative practice and interventions benefiting populations of pregnant women and their offspring. As prenatal exposure to certain environmental toxins, many of which are found in common foods and beverages, can lead to developmental deficits and malformations, common consumption practices must be appraised in current samples of pregnant women. These types of behavioral assessments play a critical role in prevention of adverse developmental outcomes that are potentially linked to a mother's intake of unhealthy substances in food, beverages and medications throughout pregnancy.

Studies investigating the impact of prenatal diet on the offspring date back to the 1920's [4], and many deleterious effects of poor diet have been reported in the literature. Reduced maternal nutrition has been associated with hypertension and altered nephrogenesis in the offspring [5,6]. Additionally, prenatal deficiencies in vitamins $\mathrm{D}$ and $\mathrm{E}$ have been associated with increased incidences of respiratory difficulties, including wheezing and asthma, in the offspring [7], and maternal vitamin D deficiencies have been related to observed hyperlocomotion in the adult rat [8]. Maternal diets high in omega-3 fatty acids may reduce sensitivity to allergies in the offspring [9], whereas methyl-donor group (vitamin B12, folic acid, and choline) supplementation during gestation is associated with increased risk of allergic inflammation in the offspring [10]. Increased cation consumption (magnesium, potassium, and calcium) was inversely related to diastolic pressure in infants [11]. These studies demonstrate that a healthy balance of nutrients play an important role in normal developmental biology.

Prenatal exposure to environmental toxins profoundly affects the developmental biology of the fetus. It is believed that exposure to toxins during the prenatal period induces developmental changes in the brain that lead to abnormal cognitive and behavioral phenotypes. Prenatal exposures to illegal drugs of abuse, such as cocaine, heroin, and methamphetamines, have been shown to impact both the developing brain and behavior [12-14]. Similarly, exposures to legal substances that are more commonly used during pregnancy, such as alcohol and nicotine, have been shown to have both short- and long-term effects on the developing baby [15-18]. Our developmental neurobiology laboratory has created two separate animal models of prenatal alcohol and nicotine exposure with compelling results $[17,18]$, suggesting their high-risk status during pregnancy. It is common practice for obstetricians to warn pregnant women of the dangers of illegal drug use while pregnant, but there is less agreement in the field as to the exact recommendation for nicotine, caffeine, alcohol and many over-thecounter (OTC) medications and prescribed drugs [19-21]. Specifically, obstetricians vary greatly in their recommendations of alcohol consumption during pregnancy with some noting that occasional light use is permitted and safe, while others suggest complete abstinence [22,23]. There are no strict guidelines in obstetrics as to whether drugs labeled by the Food and Drug Administration (FDA) as Pregnancy Category C (found to generate birth defects in animal models) are to be prescribed. Obstetricians often disagree on safe levels of caffeine consumption during pregnancy, despite its link to negative behavioral effects in the offspring [24]. Some physicians may not be aware of how pervasive intake of legal substances such as alcohol, nicotine, caffeine or OTC medication may be and, thus, fail to inform the patient of details regarding use of these common, legal substances. Behavioral assessments of consumption of these potential teratogens, in populations of pregnant woman, are thus imperative to effective preventative obstetric practice.

In addition to illegal and legal drugs, significant sources of dietary impact on the developing fetus are teratogenic substances present in unassuming ordinary foods. A wealth of new information regarding the health of our nation's food supply has been a subject of great recent interest and emerging studies have illuminated the harmful effects of methylmercury [25] and PCBs (polychlorinated biphenyls) [26], two common neurotoxic seafood contaminants present in high levels in tuna and farm-raised salmon, in offspring exposed to the toxins during gestation. Additionally, Bisphenol-A (BPA) is a potentially teratogenic xenoestrogenic monomer found in the lining of cans used for food storage. Consequences of prenatal exposure to BPAs include behavioral and reproductive abnormalities [27,28], increases in susceptibility for later developing mammary cancer [29], and alterations in reproductive systems. Understanding the rate at which pregnant woman consume certain food items is particularly important, as the list of foods that contain potential or known teratogens is growing. Researchers must strive to identify if pregnant woman are consuming toxins, and if so, preventative instructional measures should be taken by prenatal care providers to teach pregnant woman about the dangers of foods containing substances like mercury, PCBs, or BPA. 
The Hispanic population is at risk for higher fat, sodium and caloric intakes, particularly from dairy foods [30,31]. Additionally, acculturation in Hispanic populations has been thought to result in diets higher in fat and lower in fiber, with significantly lower intakes of protein, calcium, vitamin $\mathrm{A}$, vitamin $\mathrm{C}$, and folic acid [32]. Risk factors such as low socioeconomic status (SES), acculturation to US diet, and limited access to health care in Hispanic populations increase the importance of studying this population in respect to potential adverse outcomes of poor prenatal diet [33].

This article describes our findings in a sample of pregnant or perinatal women in Downey, California. We used anonymous survey measures to determine what foods, beverages, and medications pregnant women were consuming throughout gestation (Additional file 1). Our results highlight the pervasive consumption of teratogens, such as mercury and BPA, in our sample. We hope that information gleaned from our study will help inform prenatal care providers of the dangers and nature of certain consumptive habits among pregnant women.

\section{Materials and methods Questionnaire}

The Food, Beverage, and Medication Intake Questionnaire (FBMIQ) survey was designed to collect data on the prevalence and pattern of maternal consumption habits. The FBMIQ takes approximately five minutes to complete and contains questions concerning demographic information (household income, ethnicity, and age) how often and during what times during the pregnancy subjects (1) ate certain foods (e.g. fresh fruit, meat, and fast foods), (2) drank certain beverages (e.g. regular coffee, beer, and juice), and (3) ingested prescription and over-the-counter medications during pregnancy. The FBMIQ is not a complete dietary assessment; it is designed to be a short survey reflective of consumption of certain commonly consumed, relevant items. Portion size and number of servings consumed were not assessed. Based on our laboratory's work in a Fetal Alcohol Spectrum Disorders (FASD) mouse model, we were initially interested in patients' alcohol consumption during pregnancy. However, we comprised a list of potential or known teratogens as well as healthy and unhealthy practices and used a combination of these to create the survey. Non-threatening wording and reverse coding were used to maximize subject well-being and ensure accuracy.

\section{Participants and procedure}

Pregnant and recently pregnant women (36 weeks pregnant - 8 weeks post-partum) over the age of 18 were invited to complete the FBMIQ upon check in at the reception desk of obstetric and gynecology (OBGYN) offices of a private medical group in Downey, CA between
December, 2011 and December, 2012. Participants received the FBMIQ from receptionists and were informed that it was an optional nutritional survey about the habits of pregnant women. After completing the survey, participants placed completed consent forms and surveys in a blank sealable envelope provided to ensure confidentiality and alleviate concerns about anonymity. Surveys in sealed envelopes were then returned to the front desk where the receptionists collected the completed surveys. Frequency statistics for the data were presented in tables and differences between groups of age, education, and income were analyzed using Pearson correlation coefficients and nonparametric Kruskal-Wallis Tests. All statistical analyses were obtained using SPSS version 17.0. This study was conducted in strict accordance with the protocol guidelines approved by the Human Research Review Board at the University of Riverside, CA.

\section{Results}

\section{Demographics and participant information}

Participants were English and Spanish speaking pregnant or recently pregnant women (36 weeks pregnant 8 weeks post-partum) over the age of 18 who were receiving Obstetric care from a private medical group in of Downey CA. A total of 200 pregnant or recently pregnant women completed the FBMIQ. Most women (89.1\%) were aged 35 or younger, with the greatest percentage (34\%) of participants belonging to the 30-35 age group. The subject population was predominantly Hispanic (87.4\%). The remainder self-identified as $4.7 \%$ African-American, 4.2\% Asian/Pacific Islander, $2.1 \%$ White, and $0.5 \%$ Middle-eastern. Almost all of the women (95.8\%) had obtained a high school degree. Women were most likely to have completed some college without obtaining a final degree (41.9\% of respondents). Less than a third of the women (26.2\%) possessed a college or post-graduate degree. Women were most likely to have a yearly income of $\$ 25,000$ or less $(35.1 \%)$. Over two thirds (69\%) of women sampled had a yearly income of $50 \mathrm{k}$ or less. Thirteen point four percent had an income of over $\$ 75,000$ (Table 1 ).

\section{Month of pregnancy confirmation}

Nearly half $(50.5 \%)$ of the women sampled confirmed their pregnancies in the first month of gestation. That number rose to $88 \%$ by the second month. The vast majority of women (97.9\%) confirmed pregnancy sometime in the first trimester (data not shown).

\section{Foods beverages and medicinal intake during pregnancy}

Participants were asked about food, beverage, or medication intake during their pregnancies. Specifically, they were asked to identify types of foods, beverages or medications consumed and were given the option to write in 
Table 1 Demographic information of respondents

\begin{tabular}{|c|c|c|c|}
\hline Age $(n=194)$ & $\%$ Preg women & Education $(n=191)$ & $\%$ Preg women \\
\hline $18=20$ & 7.7 & Elementary & 0.5 \\
\hline $21-25$ & 20.1 & Middle school & 3.7 \\
\hline $26-29$ & 27.3 & High school & 27.7 \\
\hline $30=35$ & 34 & Some college & 41.9 \\
\hline $36-39$ & 8.2 & College degree & 17.8 \\
\hline $40+$ & 2.6 & Graduate degree & 8.4 \\
\hline Race $(n=190)$ & $\%$ Preg women & Income ( $n=171)$ & $\%$ Preg women \\
\hline White & 2.1 & $0-25 \mathrm{~K}$ & 35.1 \\
\hline Hispanic & 87.4 & $25,001 \mathrm{~K}-50 \mathrm{~K}$ & 33.9 \\
\hline African American & 4.7 & $50,001-75 \mathrm{~K}$ & 17.5 \\
\hline Asian/Pacific Islander & 4.2 & $75,001 \mathrm{~K}-100 \mathrm{~K}$ & 11.1 \\
\hline Middle-eastern & 0.5 & $100 \mathrm{~K}+$ & 2.3 \\
\hline Other & 1.1 & & \\
\hline
\end{tabular}

Sample characteristics of respondents. Values for Age, Education, Race, and Income are listed as percentages for number of pregnant women. $(\mathrm{n}=171-194)$.

choices that were not explicitly listed in the survey (See Tables 2, 3, 4). Participants were also asked to report the frequency and trimester specificity of consumption (Table 5).

\section{Food consumption habits}

Participants were asked whether they ate chicken, beef, or pork during their pregnancies (fish consumption patterns were assessed in a different survey section, and thus fish was excluded from the meat category). Almost all of the women sampled (99.5\%) ate meat some time during their pregnancies, and almost two-thirds $(66.1 \%)$ ate meat during all three trimesters (Table 3, Table 5). Chicken was the most frequently reported meat consumed $(98.5 \%)$, followed by beef ( $84 \%)$, then pork (52\%). Most women $(81.9 \%)$ consumed meat at least once a week, with $30.4 \%$ consuming meat at least four times a week (Figure 1, Table 2, Table 5).

Most participants $(73.9 \%)$ consumed fish during pregnancy. The most commonly consumed type of fish was tuna (52\%), followed by tilapia (34.2\%) and salmon $(25.5 \%)$. Less than a quarter of the women $(19.9 \%)$ also reported eating other kinds of fish or shellfish, with shrimp being the most frequently written in option. Most women ate fish less than once a week $(80 \%)$, and nearly a third of women (31.2\%) ate fish during all three trimesters (Figure 1, Table 2, Table 5).

All women reported eating fresh fruit during their pregnancies. Bananas were the most commonly eaten fruit $(95.4 \%)$, followed by oranges (88.8\%), and apples (88.3\%) (Figure 1, Table 2). Women also reported eating other fruits such as strawberries, pears, watermelon, and grapes. Two thirds (65.8\%) of women ate fruit at least four times a week. Although the majority of women (77\%) reported consuming fruit during all three trimesters of their pregnancies, only a third of the women (31.1\%) ate the recommended amount of more than one serving of fruit per day (Table 5).

The majority of participants (73.9\%) reported consuming canned foods during their pregnancies, with 11.9\% reporting consumption frequencies of four or more instances a week. Nearly half of sampled women (48.9\%) reported eating canned foods during all three trimesters of their pregnancies (Table 5). Canned fruits/vegetables and soup were most commonly consumed $(52.3 \%$ and $41.6 \%$ of women, respectively) followed by canned tuna (41.6\%) (Figure 2; Table 2).

Almost all women (97.5\%) reported eating high-sugar desserts during their pregnancies. Ice cream was the favored dessert: $82.7 \%$ reported eating ice cream. Additionally, $70.1 \%$ reported eating baked desserts, 65\% reported eating chocolate, and 3.6\% reported eating other desserts, such as candy or frozen dessert beverages during their pregnancies. Most women (76.2\%) consumed desserts between one time per month and three times per week. Over half (53.2\%) reported eating sugary desserts throughout their pregnancies (Figure 2, Table 2, Table 5).

Nearly all of the women (96\%) reported eating fast foods during their pregnancies, with burgers being the most commonly consumed item (85.2\%), followed by french fries $(77.9 \%)$, chicken products $(53.6 \%)$, and other fast foods such as Mexican fast foods and chicken salads. Consumption patterns for fast food intake were varied: 19.1\% reported only eating fast foods 1-3 times during their entire pregnancies, $47.5 \%$ reported eating fast foods 1-3 times per month, and $25.7 \%$ reported eating fast foods 1-3 times per week. Only 5.5\% of women reported eating fast food more than four times per week during 
Table 2 Food consumption habits

\begin{tabular}{|c|c|}
\hline Food consumed & $\%$ Preg wome \\
\hline Any meat & 99. \\
\hline Chicken & 98. \\
\hline Beef & 8 \\
\hline Pork & 5 \\
\hline Any fish & 73. \\
\hline Tuna & 5 \\
\hline Tilapia & 34. \\
\hline Salmon & 25. \\
\hline Other fish & 19. \\
\hline Fresh fruit & 10 \\
\hline Banana & 95. \\
\hline Oranges & 88 \\
\hline Apples & 88. \\
\hline Other fresh fruit & 40. \\
\hline Any canned foods & 73. \\
\hline Canned fruits/veggies & 52. \\
\hline Canned soup & 41. \\
\hline Canned tuna & 41. \\
\hline Sugary desserts & 97. \\
\hline Ice cream & 82. \\
\hline Baked desserts & 70. \\
\hline Chocolate & 6 \\
\hline Other desserts & 3. \\
\hline Fast foods & $y$ \\
\hline Burger & 85. \\
\hline French fries & 77. \\
\hline Chicken products & 53. \\
\hline Others fast foods & 7. \\
\hline
\end{tabular}

Number of women who reported consuming a given food during pregnancy. Values are listed as percentages for number of pregnant women. $(n=195-200)$.

their pregnancies. Forty-three percent of women reported eating fast foods during all three trimesters (Figure 2, Table 2, Table 5).

\section{Beverage consumption habits}

All women reported drinking water, with $96 \%$ reporting consumption of bottled water, $36.9 \%$ consuming homefiltered water, and $12.1 \%$ consuming tap water during their pregnancies (Figures 1 and 2, Table 3).

Most women (95.4\%) drank milk during their pregnancies. Of those women, $79 \%$ drank low-fat milk, 20.9\% drank whole milk, $6.7 \%$ drank organic milk, 3.9\% drank skim milk, and $8.6 \%$ drank "other" milk, with 3.6\% identifying "other" as soy milk. Two-thirds of women (66.6\%) drank milk at least 4 times per week. Most
Table 3 Beverage consumption habits

\begin{tabular}{|c|c|}
\hline Beverage consumed & $\%$ Preg women \\
\hline Any water & 100 \\
\hline Bottled water & 96 \\
\hline Home filtered water & 36.9 \\
\hline Tap water & 12.1 \\
\hline Other water & 1.5 \\
\hline Any milk & 95.4 \\
\hline Lowfat & 79 \\
\hline Whole & 20.9 \\
\hline Other milk & 9.2 \\
\hline Organic & 6.7 \\
\hline Skim & 4.1 \\
\hline Any juice & 94.9 \\
\hline Orange juice & 76.8 \\
\hline Apple juice & 69.1 \\
\hline Juice blends & 41.2 \\
\hline Other juice & 12.9 \\
\hline Any caffeine & 80.1 \\
\hline Colas & 60.2 \\
\hline Coffee & 45.5 \\
\hline Tea & 29.8 \\
\hline Other caffeinated beverages & 2.1 \\
\hline Energy drinks & 1.5 \\
\hline Decaffeinated beverages & 22.5 \\
\hline Any alcohol & 5.8 \\
\hline Beer & 1.6 \\
\hline Wine & 4.7 \\
\hline Mixed drinks & 1.1 \\
\hline Shots/liquor & 0.5 \\
\hline
\end{tabular}

Number of women who reported consuming a given beverage during pregnancy. Values are listed as percentages for number of pregnant women. ( $n=188-199)$.

(71.4\%) drank milk during all three trimesters. (Figure 1, Table 3, Table 5).

A total of $94.9 \%$ of women reported drinking juice during their pregnancies. Orange juice was the most commonly consumed juice (76.8\%) followed by apple juice (69.1\%), juice blends (41.2\%) and other juices (12.9\%) such as cranberry or pineapple juice. Most women (85.5\%) reported drinking juice at least once per week, and over two thirds (68\%) reported drinking juice throughout their pregnancies (Figure 1, Table 3, Table 5).

Participants were asked to report whether they had consumed regular coffee, regular tea, colas, or decaffeinated beverages during their pregnancies. The majority of women sampled $(80.1 \%)$ had consumed caffeinated beverages during their pregnancies (Figure 3, Table 3). Colas were the most popular caffeinated beverage (60.2\% 
Table 4 Medication/vitamin consumption

Substance consumed $\%$ Preg women

Over-the-counter meds

45.3

Acetaminophen

38

Cough/cold meds

Ibuprofen

Aspirin

Decongestants

Prescription meds

Prenatal vitamins

Morning sickness medications

Pain medications

Antidepressants

Number of women who reported consuming vitamins or medications during pregnancy. Values are listed as percentages for number of pregnant women. $(n=192-194)$.

of all pregnant women), followed by coffee (45.5\%) and tea $(29.8 \%)$. Forty point four percent of women who reported drinking caffeinated beverages did so throughout their pregnancies, while nearly a fifth (18.6\%) only consumed caffeine during the mid- and later parts of their pregnancies (Figure 3, Table 3, Table 5).

Out of 190 women who responded to any questions about alcohol usage, 5.8\% reported drinking alcohol sometime during their pregnancies (Figure 3, Table 3). When asked about the type of alcohol consumed, these women reported drinking beer, wine, or champagne. Consumption of mixed drinks and hard liquor was less common, with only a couple of women reporting this type of alcohol use (Figure 3, Table 3).

Number of alcohol units consumed during pregnancy was relatively low; most women (nine out of the ten who reported gestational alcohol use) only consumed $1-3$ units of alcohol (bottles, glasses, or shots) during the entire pregnancy. The exception was one participant who reported an alcohol consumption rate of 1-3 alcohol units per month during the first trimester (Table 5).

Alcohol usage typically occurred during the first trimester, with the exception of four participants, who reported alcohol usage in the second or third trimester (Table 5).

\section{Consumption of vitamins and medications}

Most women reported consuming prenatal vitamins during their pregnancies $(83.4 \%)$. Eight point eight percent of women reported taking prescription morning sickness medicine, and 3.6\% reported taking prescription pain medications. Acetaminophen was the highest used over-the-counter medication (38\%). A small number of women reported taking aspirin (1.6\%), ibuprofen (3.1\%),

Table 5 Frequency and trimester of consumption

\begin{tabular}{|c|c|c|c|c|c|c|c|c|c|c|c|c|}
\hline \multirow[t]{2}{*}{ Substance consumed } & \multicolumn{5}{|c|}{ Frequency (displayed as \% of women reporting intake) } & \multicolumn{7}{|c|}{ Trimester (displayed as \% of women reporting intake) } \\
\hline & 1-3 entire preg. & $1-3 /$ month & 1-3/week & 4-6/week & 7+/week & 1 & 2 & 3 & $1,2,3$ & 1,2 & 2,3 & 1,3 \\
\hline \multicolumn{13}{|l|}{ Food } \\
\hline Meat & 5.2 & 12.9 & 51.5 & 22.2 & 8.2 & 9.9 & 9.4 & 3.6 & 66.1 & 2.6 & 7.8 & 0.5 \\
\hline Fish & 41.4 & 38.6 & 16.6 & 2.8 & 0.7 & 12.3 & 27.5 & 8.7 & 31.2 & 4.3 & 14.5 & 1.4 \\
\hline Fruit & 2.6 & 3.6 & 28 & 34.7 & 31.1 & 6.3 & 4.2 & 1.6 & 77 & 3.1 & 7.3 & 0.5 \\
\hline Canned foods & 28 & 31.5 & 28.7 & 9.8 & 2.1 & 16.5 & 11.5 & 5 & 48.9 & 6.5 & 10.1 & 1.4 \\
\hline Sugary desserts & 10.6 & 39.2 & 37 & 10.1 & 3.2 & 8.1 & 14 & 9.7 & 53.2 & 2.2 & 12.9 & 0 \\
\hline Fast foods & 19.1 & 47.5 & 25.7 & 5.5 & 2.2 & 8.9 & 15.1 & 10.1 & 43 & 7.8 & 15.1 & 0 \\
\hline \multicolumn{13}{|l|}{ Beverage } \\
\hline Water & 1.5 & 0.5 & 3.1 & 6.7 & 88.2 & 5.8 & 3.2 & 0.5 & 85.8 & 2.1 & 2.1 & 0.5 \\
\hline Milk & 2.2 & 8.2 & 23 & 35.5 & 31.1 & 6 & 4.4 & 1.1 & 71.4 & 4.9 & 11.5 & 0.5 \\
\hline Juice & 2.8 & 11.7 & 35.8 & 30.7 & 19 & 7.4 & 6.3 & 2.8 & 68.8 & 6.3 & 8 & 0.6 \\
\hline Caffeine & 14 & 30.7 & 40.7 & 11.3 & 3.3 & 10.9 & 15.4 & 8.3 & 40.4 & 5.1 & 18.6 & 1.1 \\
\hline Beer/wine & 88.9 & 11.1 & 0 & 0 & 0 & 50 & 30 & 10 & 0 & 10 & 0 & 0 \\
\hline Mixed drinks/liquor & 100 & 0 & 0 & 0 & 0 & 100 & 0 & 0 & 0 & 0 & 0 & 0 \\
\hline \multicolumn{13}{|l|}{ Medication } \\
\hline Over-the-counter & 53.7 & 31.3 & 7.5 & 3 & 4.5 & 19.3 & 21.7 & 13.3 & 28.9 & 6 & 7.2 & 3.6 \\
\hline Prescription & 7.1 & 5.3 & 1.8 & 8.8 & 76.1 & 10.9 & 2 & 2 & 72.8 & 6.1 & 4.1 & 2 \\
\hline
\end{tabular}

Frequency of consumption for foods, beverages, and medications and with trimester for which frequency data is applicable. All values are percentages of women who reported intake of a given item. Frequency values are listed as percentages for number of women who reported consuming a given item 1-3 times during their entire pregnancy, 1-3 times per month, 1-3 times per week, 4-6 times per week, or 7 or more times per week ( $n=10-194)$. Trimester values are listed as percentages for number of women who reported consuming a given item during the first, second, or third trimester only, all three trimesters, or two of the three trimesters. $(n=10-200)$. 


\section{Healthy eating habits}

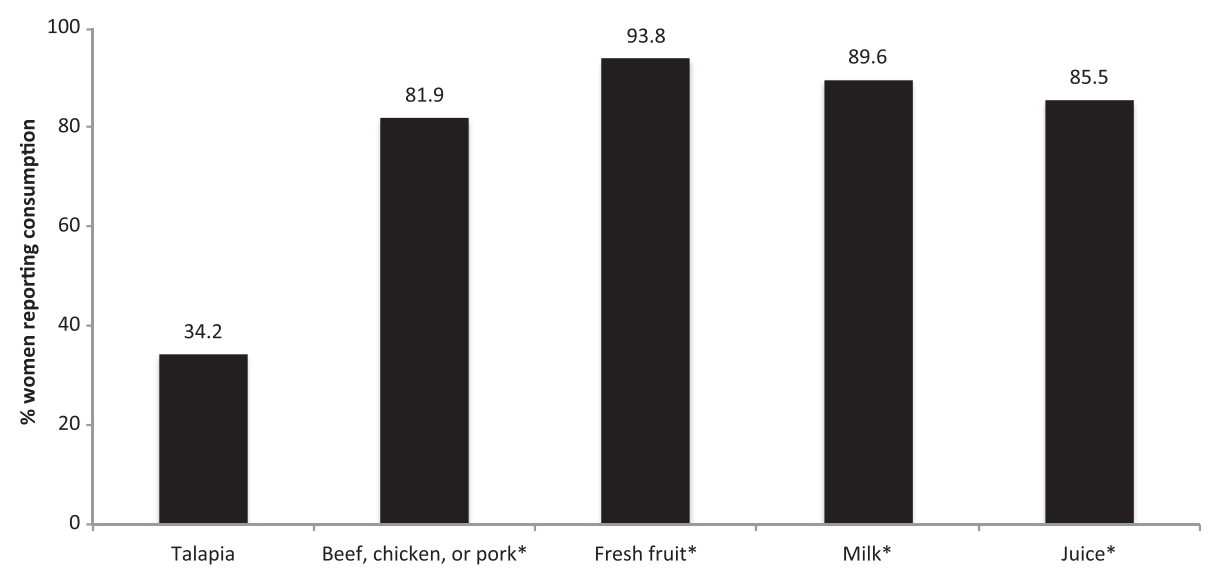

Figure $1 \mathrm{Graphical}$ depiction of percentages of women who reported eating healthy foods during their pregnancies. Asterisks indicate percentages of women who ate or drank a given food or beverage at least once per week. $(n=196-200)$.

decongestants (1.6\%) and cough and cold medications (4.2\%; Table 4) during their pregnancies.

\section{Demographic factors and risky consumption habits}

Pearson correlation coefficients between demographic factors (age, education, and income) and dietary risk factors (consumption of tuna, salmon, canned foods, sugary desserts, fast foods, tap water, caffeine, and alcohol) were calculated. Women with lower income were more likely to have reported eating any canned foods during their pregnancies $(\mathrm{r}=-0.143 ; \mathrm{p}<0.05 ; \mathrm{n}=191)$. No other significant correlations were found.

Non-parametric Kruskal-Wallis Tests were performed to explore differences in consumption frequencies for fish, canned foods, sugary desserts, fast foods, caffeinated beverages, and alcohol with grouping variables of age, education, and income. Kruskal-Wallis tests revealed no significant differences in consumption frequencies among groups.

\section{Discussion}

\section{Healthy eating and drinking}

Consumption of beef, chicken, tilapia, fruit, bottled or filtered water, milk, juice, and decaffeinated beverages were considered to be generally safe and healthy for the developing fetus. While recent risk factors associated with meat products have been described [34], the beneficial dietary supplementation of these choline-rich foods has been judged to outweigh the risks associated with hormone and antibiotic levels in certain meat and diary products. Likewise, high-sugar content in certain types of juices may be associated with the onset of gestational

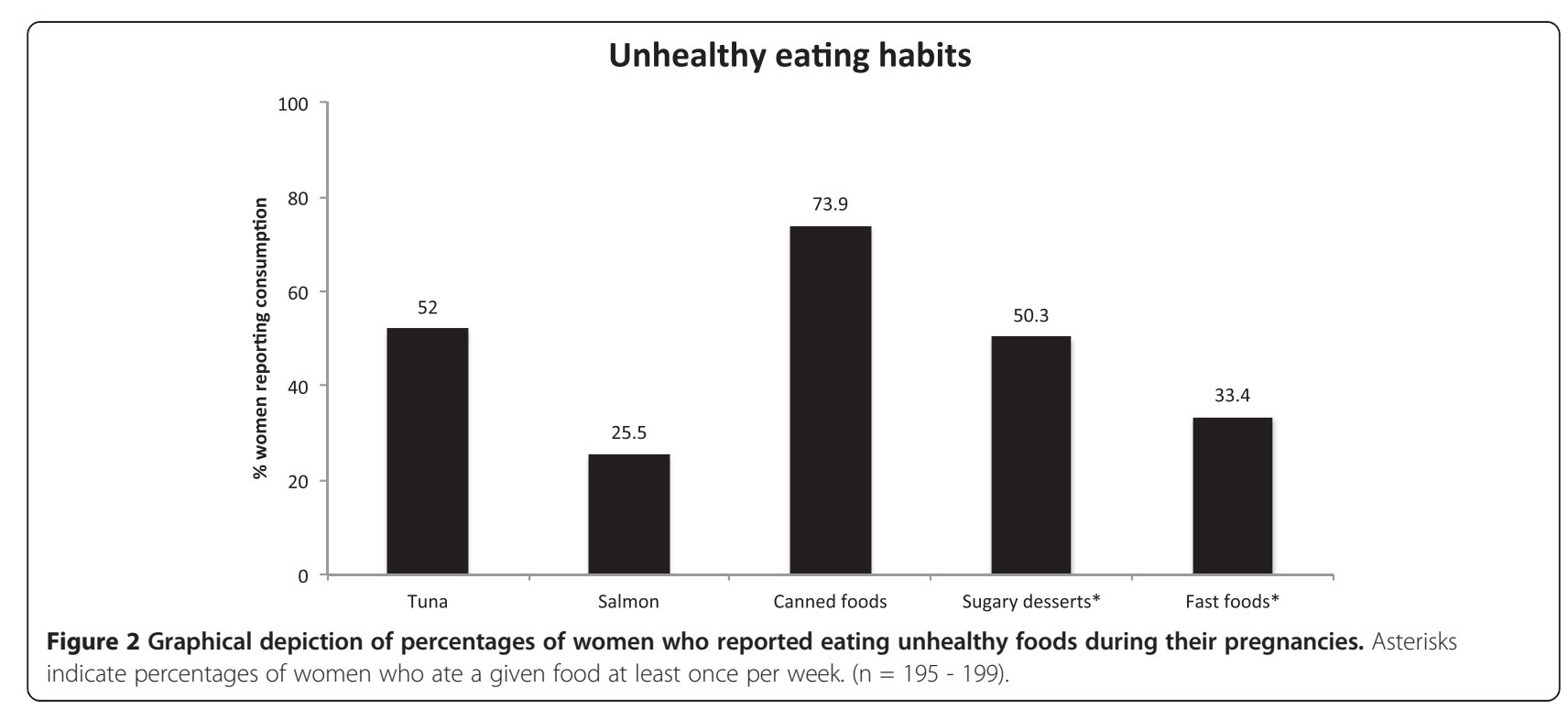




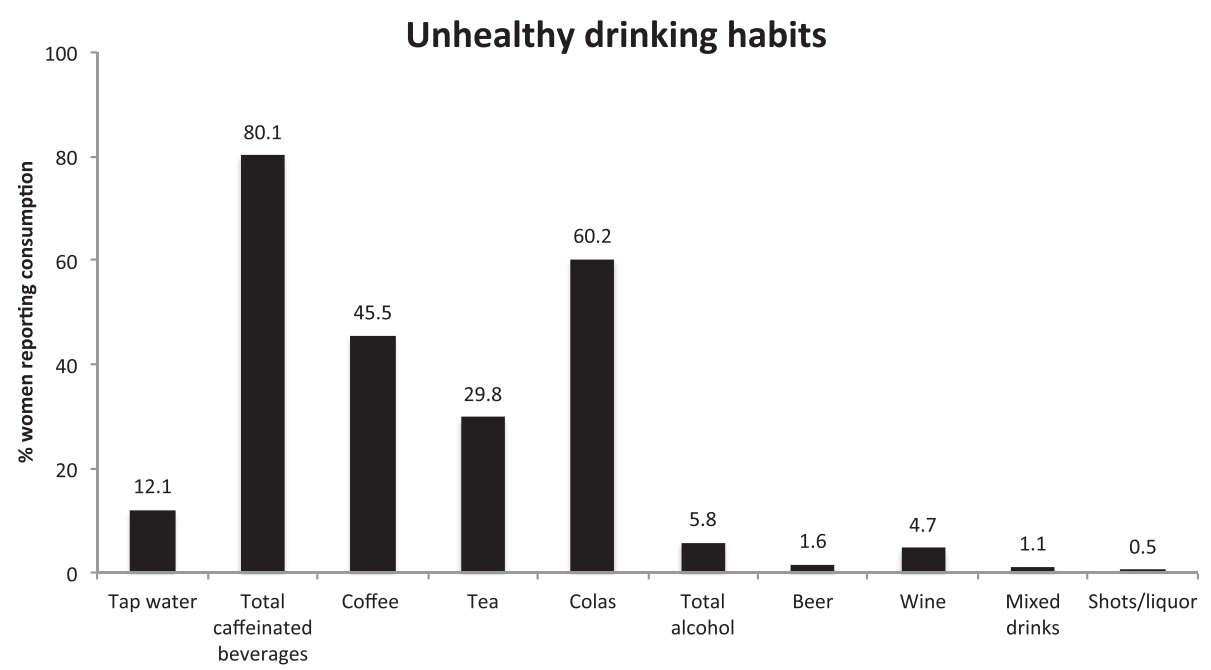

Figure 3 Graphical depiction of percentages of women who reported drinking unhealthy beverages during their pregnancies. $(n=188-199)$.

diabetes; however, the vitamins and nutrients in juices merit their inclusion in the healthy drinking category. The American Dietetic Association recommends, for pregnant women, a healthy diet in accordance with the Dietary Guidelines for Americans (2005) including a variety of daily grains, milk products, fruits, vegetables, and iron-rich meats $[1,35]$. The majority of pregnant women reported eating healthy foods including tilapia, beef, chicken, or pork, fresh fruit, milk, and juice during their pregnancies. The frequencies at which women in our sample ate these foods did not strictly adhere to daily recommendations. Only a third of women, for example, reported eating fruit 7 or more times a week.

\section{Unhealthy consumption habits}

Consumption of tuna, salmon, canned goods, sugary desserts, fast foods, and drinking of tap water, caffeinated beverages, and alcoholic beverages during pregnancy have been deemed unhealthy due to the appearance of environmental toxins found to have harmful effects in the developing offspring.

\section{Tuna}

The bioaccumulation of methylmercury $(\mathrm{MeHg})$ in marine life, particularly tuna, presents a threat for developing fetuses whose mothers frequently eat this fish during their pregnancies, particularly because it is thought that mercury accumulates more readily in the fetal brain than in the maternal brain, interrupting patterns of cell fate, proliferation, migration, and neural outgrowth [36-38]. Because young children cannot metabolize mercury at the same rates as adults, exposure through either maternal or childhood consumption is of great concern. Even low levels of tuna consumption in children can readily result in blood mercury levels that exceed the heath limit [39].
Epidemiological studies with cohorts from fish-eating populations have found that prenatal exposure to methylmercury has been associated with a myriad of developmental deficits involving attention, verbal learning, visuo-spatial and motor function, and delayed performance $[25,40]$. The FDA reports that mean mercury levels of tuna range from $0.128 \mathrm{ppm}$ (light canned tuna) to $0.689 \mathrm{ppm}$ (big-eye tuna). However, mercury levels are highly variable and the FDA reported canned tuna to contain as much as $0.889 \mathrm{ppm}$ for light canned tuna, and $1.816 \mathrm{ppm}$ for big-eye tuna [41]. Currently, the FDA and the Environmental Protection Agency (EPA) recommend that pregnant women eat no more than six ounces of tuna per week. However, tests performed by Consumer Reports on new samples of white tuna revealed that eating only 2.5 ounces of any of the new samples of white tuna would cause pregnant women to exceed the daily mercury levels that the EPA considers safe [42]. Worryingly, more than half of the women $(52 \%)$ in this study reported consuming tuna during their pregnancies, suggesting that pregnant woman are generally not aware of risk associated with tuna consumption.

\section{Salmon}

Polychlorinated biphenyls (PCBs) are lipophilic compounds found in fatty tissues of marine life feeding in contaminated waters. Staggering levels of PCBs have been found in farmed salmon compared to their wild-type counterparts, and contaminated commercial salmon feed has lead to the bioaccumulation of these dangerous compounds [43]. Prenatal exposure of PCBs has been linked to lower birth weights, smaller head circumferences, and abnormal reflex abilities in newborns, as well as to mental impairment in older children [26,44-46]. The FDA limits limiting PCB residues in fish to $2 \mathrm{ppm}$ [47]. Although epidemiological studies seem to suggest $\mathrm{PCB}$ exposure is 
related to poor outcomes in neurodevelopment, the precise exposure patterns leading to these deficits have not been characterized [46]. However, a recent metaanalysis of 12 European studies found an association between fetal growth and PCB exposure at low, clinicallyrelevant levels [48].

More than a quarter of the women (25.5\%) surveyed reported consuming salmon, a commonly ingested source of PCBs. Although prenatal fish intake, including tuna and salmon, can be a good source of Docosahexaenoic acid (DHA), a fatty acid thought to be beneficial in development, fetuses may be at risk for adverse outcomes, and pregnant women should be advised to be selective about which fish they choose to consume, or seek supplementation with fish oil.

\section{Canned foods}

Bisphenol A (BPA) found in the lining of metal cans used for food represents a danger to developing fetuses whose mothers consumed a diet high in canned foods. $\mathrm{BPA}$ has received recent attention as a controversial ingredient in child sippy cups, baby bottles, and reusable water bottles, leading to a ban by the FDA on the use of the plastic additive in sippy cups and baby bottles $[49,50]$. Perhaps less well-known are the dangers of BPA exposure in consuming canned foods. Leaching of BPA from the epoxy resin of metallic food cans has been demonstrated in many studies [49,51,52]. Prenatal exposure to BPA has been found to be associated with higher externalizing scores (hyperactivity and aggression) in two-year-old females and reproductive effects in rodent models $[27,28]$. The majority of women surveyed (73.9\%) reported consumption of canned foods during their pregnancies, with $11.9 \%$ reporting consumption at least 4 times a week, suggesting women may not be aware of the dangers of BPA exposure from canned foods. Although epidemiological studies concerning prenatal BPA exposure are lacking, animal studies warn of the detrimental effects of BPAs. Children of pregnant women maintaining a diet high in canned foods are at risk for adverse postnatal outcomes. This study has found that low income is inversely correlated with canned food consumption, suggesting that women of low SES in particular may be especially at risk.

\section{Sweet desserts}

Frequent consumption of sugary desserts during pregnancy may contribute to increased likely-hood of gestational diabetes mellitus (GDM), a condition of glucose intolerance that has been implicated in many pregnancy problems including macrosomia, large for gestational age (LGA) infants, and increased rates of cesarean delivery [53-57]. Hispanic women in particular are two and half times more likely than non-Hispanic whites to suffer from GDM [58]. Increased sugar intake among pregnant adolescents has been linked to maternal gestational diabetes and LGA infants $[57,59,60]$, and problems with gestational glucose control have been associated with neural tube defects [61]. On the other hand, carbohydrate restriction has been shown to aid in maternal glycemic control, alleviating some of the adverse pregnancy outcomes seen in patients with GDM [54]. More than a third of women (37\%) ate sweet desserts more than one time per week, and $13.3 \%$ ate desserts more than 4 times per week. Children born of women maintaining a diet high in sweet desserts are at risk of macrosomia and postnatal obesity. Of course, women with GDM should be advised to closely monitor their sugar intake during pregnancy.

\section{Fast foods}

The past decades have seen an insurgence of reliance upon high-energy, low nutrient foods that correlate with rising rates of obesity. A prenatal diet high in fast food represents a possible danger to the developing fetus, as these foods usually contain high levels of fat and salt. Maternal diets high in fat have been associated with increased likelihoods of postnatal diet-induced obesity in offspring, and have the potential to influence epigenetic markers leading to altered postnatal gene expression and eating behavior [62,63], Prenatal diets high in sodium levels have been linked to decreased gestational weight gain and an increased responsiveness to stress in adults $[64,65]$. More than a quarter of women surveyed $(25.7 \%)$ report eating fast foods at least once a week during their pregnancies. An alarmingly high percentage of surveyed women reported consuming fast foods more than four times per week (7.7\%), and are at heightened risk for adverse fetal effects of high maternal salt and fat diets. Additionally, Hispanic populations are at risk for higher fat intakes from dairy foods [30]. Based on the literature, prenatal advising should stress the importance of eating a healthy diet low in these energy-dense, nutrient low foods so as to lower future generations' risk of obesity and stress conditions.

\section{Tap water}

Drinking water has been found to have levels of many prenatal toxins including trihalomethanes, and certain drinking water disinfection by-products (dibromoacetic acids, or DBAs). The Drinking Water Quality Report for Downey, CA warns of 20 different pollutants in the city's water, with eight chemicals existing at concentrations that exceed the health guidelines set by federal and state agencies: tri - and tetro-chloroethylene (DBAs), alpha particle activity, arsenic, radium 228, lead, radium 226, and combined radium [66]. Many women in our sample (12.1\%) reported drinking tap water during pregnancy, 
suggesting risk for exposure to many of these dangerous contaminants. Contaminants such as arsenic and DBA, and radium 226 have been found to result in central nervous system defects, oral cleft defects, and neural tube defects, and small for gestational age births, and risks for fetal death [67-69]. Women drinking tap water in areas where contamination is exceptionally high, as in Downey, CA, are at risk for adverse outcomes resulting from prenatal exposure to certain chemicals. Pregnant women should instead be encouraged to drink filtered or bottled water.

\section{Alcohol}

The spectrum of disabilities associated with prenatal alcohol exposure is termed FASD (Fetal Alcohol Spectrum Disorders). The prevalence of FASD has been difficult for researchers to ascertain. Some studies have found that anywhere from 0.5 - 2 cases per live births [70]. One study found as high as 1 per 100 live births when the full range of FASD was taken into account [71]. There is a large amount of data characterizing the severe complications in children who were exposed prenatally to alcohol. Children born with FASD often exhibit abnormal craniofacial features and have a litany of cognitive impairments including learning disabilities, decreased intelligence, decreased reaction time, slow sensory processing speed, language dysfunction, and behavioral disorders that are direct results of nervous system injury [72-75].

\section{Self-reporting of maternal drinking}

Information on the prevalence and pattern of maternal drinking has been notoriously difficult to ascertain. One possible diagnosis tool is the detection of biomarkers at birth. FAEEs (fatty acid ethyl esters) accumulate in the meconium after alcohol exposure in the first and second trimester [76]. Such methods have been useful in detecting alcohol usage in at-risk subjects at birth, but are impractical for use in a comprehensive assessment of average alcohol usage during the entire gestational period. Thus, although biological methods of measuring alcohol usage during pregnancy have been developed, self-reports of maternal drinking remain the most effective methodology for maternal drinking prevalence assessments.

Ten women (5.8\%) surveyed in Downey, CA reported drinking some time during their pregnancies. This number is slightly lower than national estimates of maternal drinking from the Centers for Disease Control and Prevention (CDC; $7.6 \%$ of pregnant women surveyed) [77]. Differences in rates may be due to the demographic make up of our sample. Maternal drinking rates are highest in white populations of older individuals (35-44 years of age) [77]. This study examined a population of relatively young (89.2\% under 35$)$ women who were predominantly Hispanic (87.4\%). Methodological differences may also be at play. CDC data is obtained from the Behavioral Risk Factor Surveillance System (BRFSS), a telephone survey system that asks women to report alcohol usage within the last 30 days. Conversely, the current study surveys participants in late pregnancy or early postpregnancy periods, when subjects might have difficulty remembering nutritional habits from early pregnancy. The chance of reporting inaccurate information from early pregnancy rises with the passing of time, and the stigma associated with maternal drinking might influence participants to deny usage if recall was suspect. However, despite the difficulties in obtaining accurate data through selfreport, administration of a survey instrument to this population of women is the best way to obtain information regarding behaviors during pregnancy.

Methyl donors, prenatal vitamins, and alcohol consumption Research has consistently shown that intake of folate, choline, and other methyl donors are integral to the healthy development of the fetus. Specifically, the metabolism of these nutrients provides methyl groups in one-carbon methylation pathways [78]. Disruptions in one-carbon metabolism may result in decreased cognitive abilities [79] and serious birth defects [80]. Folic acid is an important contributor of methyl groups for pregnant women. A recent study has suggested that intake of prenatal vitamins may reduce the risk of autism [81]. Fortunately, intake of prenatal vitamins is fairly common: $83.4 \%$ of women report supplementing their diets with prenatal vitamins. Additionally, consumption of choline-rich foods was high: all women reported eating meat sometime during their pregnancies, and most reported eating meat at least once per week.

Because prenatal alcohol has been found to disrupt one-carbon metabolism, diets deficient in methyl donors may exacerbate the harmful effects of prenatal alcohol. Although women seem to be making an effort to curb their alcohol usage after recognizing their pregnancy, the number of women who report drinking during the first trimester is worrying (half of the ten women who reported drinking). Research has shown early pregnancy to be especially vulnerable to the teratogenic effects of alcohol, as deficiencies in methyl donor groups in the first month have been shown to result in neural tube birth defects in offspring [78].

\section{Caffeine usage}

Caffeine is a xanthine alkaloid that can be found in coffee, sodas, energy drinks, and tea. Studies labeling caffeine as a teratogen date back to the late 1960s [82], and in 1980, the FDA advised limiting intake of caffeine during pregnancy, noting the substance's association with fetal mortality, birth defects, and decreased birth 
weights $[83,84]$. The American Pregnancy Association recommends $150-300 \mathrm{mg}$ as a safe daily dose of caffeine, although this is only based upon studies concerning risk of miscarriage [85]. Published epidemiological studies noting the impact of prenatal caffeine on child behavioral and cognitive effects have been somewhat scarce and inconclusive [24,86-88]. Animal studies, however, have found developmental delays, abnormal neuro-motor activity, and neurochemical disruptions, with some effects persisting until adulthood (for review, [89]). High levels of coffee consumption have been linked to fetal death after the second trimester $[90,91]$.

Caffeine has been consistently linked to abnormal motor activity and motor development $[87,88]$. A majority of the subjects (80\%) reported drinking caffeinated beverages during their pregnancies, with $14 \%$ of women reporting consumption of more than 4 caffeinated beverages per week. These numbers suggest that some children may be at risk for preventable persistent aberrations in neurochemistry and motor development.

\section{Intake of over-the-counter and prescription medication}

The number of women taking over-the-counter and prescription medications during pregnancy has increased within the past few decades [92]. A small number of women report using aspirin (1.6\%) and ibuprofen (3.1\%) during pregnancy. Animal experiments have implicated aspirin and ibuprofen, both cyclooxygenase inhibitors, in a number of adverse fetal effects including physical malformations [93,94] and postnatal cognitive deficits [95].

A small number of women (3.6\%) reported taking prescription pain medications during pregnancy. Fetal effects as a result of prenatal opioid exposure are poorly understood, but seem to be related to poor developmental outcomes [96]. Case control studies have made associations between prenatal use of opioid analgesics and congenital heart defects, the primary factor in birthdefect related infant mortalities [97].

Less than $10 \%(8.8 \%)$ of women reported using prescription anti-nausea medications. Use of anti-nausea medications have been found to be associated with acute non-lymphoblastic leukemia [98] although some FDA pregnancy category B drugs (considered safe in pregnancy) such as Zofran, are often prescribed for pregnancy related morning sickness. Our study did not differentiate between the pregnancy categories of anti-nausea medications used.

A paucity of conclusive research on the effects of prenatal exposure to prescription drugs may have led to the assumption that they are safe to prescribe. However, studies highlighting the possible dangers of prescription drugs, as well as some over-the-counter medications, suggest otherwise. Use of opioid analgesics and category $\mathrm{C}$ and above anti-nausea medications put pregnant women at risk for aversive offspring outcomes. In any case, women should be properly informed as to the possible dangers of prenatal exposure to these medications.

\section{Changing consumption patterns after recognition of pregnancy}

Reports of patterns of consumption, that is, information about the amount of a substance consumed and the period during pregnancy in which it was consumed, can provide a window into commonplace beliefs about what habits are healthy during gestation. For example, most women who consumed alcohol during pregnancy reported doing so only in the first trimester. Given that a significant number of women (48.6\%) confirm their pregnancies halfway through the trimester, it is likely that these women who report drinking during the first few months are doing so before realizing they are pregnant. In contrast, the majority of women consuming caffeine during pregnancy report continuing their consumption of these beverages well into their second and third trimesters, suggesting that caffeine is not commonly regarded as harmful to the unborn fetus. The implications of this research are two fold: firstly, women of childbearing age hoping to conceive should be advised to eliminate all alcohol consumption, as effects of maternal drinking have dire consequences in the first trimester when the mother may not know she is pregnant. Additionally, every effort should be made during clinical prenatal care visits to inform pregnant women of the harmful effects of environmental toxins that can be readily transferred to the fetus as a result of uninformed, unhealthy consumption habits.

\section{Study limitations}

Several problems exist in attempt to extract such sensitive data (such as gestational consumption of alcohol) from participants, with underreporting and recall bias as major hurdles to accurate data collection. The uncomfortable nature of questions concerning maternal drinking may lead to extensive underreporting of alcohol consumption. Underreporting is suspected to be a major issue in the field [99]. Nevertheless, self-reports of maternal drinking remain the most effective tool for sensitive assessments.

Unfortunately, our data was limited to women who agreed to participate in our survey, and we were unable to randomize samples. As survey distribution was offsite at a private medical group in Downey, CA, we do not have information on percentages of women who were not included in the survey. Small sample sizes for other ethnicities such as Whites, African Americans, and Asians made cross-ethnic studies unfeasible. Similarly, we were unable to conduct certain analyses on a low but significant population of women reporting alcohol use 
during pregnancy. The FBMIQ was designed to be a short 5-minute study assessing percentages of women who may be at risk for certain unsafe consumption habits and as such it does not represent a complete dietary intake assessment. The scope of the survey limited us to frequency and trimester information only in major food categories, thus we were unable to report frequencies of subcategory items (prenatal vitamins versus prescription pain medication, for example). Additionally, although many substances in foods and beverages are thought to be teratogenic at high levels, specific dosages and patterns of exposure leading to adverse outcomes are not yet known. Developmental deficits as a result of prenatal exposure to environmental toxins at clinicallyrelevant levels, along with reviews and meta-analyses of the current literature, are important areas of future research that will help inform future prenatal guidance protocols.

\section{Conclusions}

The current study analyzes dietary habits of pregnant or recently pregnant women in Downey, CA, with particular emphasis on consumption intake of substances thought to be teratogenic in nature. Our main findings are summaries as percentages of women reporting consumption of unhealthy foods and beverages during pregnancies. For example, we found high numbers of Hispanic pregnant women consumed methylmercury through tuna, PCBs through salmon consumption, BPA through canned goods, DBAs containing tap water, caffeine containing beverages, and alcohol containing beverages during pregnancy. We also found that large percentages of pregnant Hispanic women reported eating high sugar sweet desserts and high fat and salt fast foods more than once a week. A small number of women reported the use of certain nonrecommended over-the-counter medications such as aspirin and ibuprofen, as well as prescription medications with unsafe FDA pregnancy categories. These data reflect a remaining risk in certain populations for adverse outcomes in fetal development. Fortunately, a majority of the women surveyed report taking prenatal vitamins, which aid in the prevention of many neural tube defects. Additionally, percentages women reporting healthy consumption habits were generally high. In summary, our findings in a population of predominantly Hispanic women suggest high levels of consumption of substances that serve as potential teratogens to unborn children. Because we have not surveyed other populations of pregnant women, we do not know whether this is something unique to Hispanic women, or ubiquitous among women of multiple ethnicities. However, it is clear that prenatal medical professionals should discourage the consumption of dangerous foods, beverages, and medications that women commonly report consuming during pregnancy.
In light of our consumption data here, prenatal professionals, including but not limited to OBGYNs, should be encouraged to instruct their pregnant patients about the dangers of hidden teratogens in our food supply, including but not limited to methylmercury and PCBs in fish, caffeine and alcohol in beverages and BPA in canned goods.

\section{Additional file}

Additional file 1: Anonymous (no names) survey.

\section{Competing interest}

The authors declare that they have no competing interest.

\section{Authors' contributions}

SS participated in the coordination of the study, performed the statistical analysis, helped draft the format of the survey, and helped draft the manuscript, figures, and tables. GP helped conceive the initial design of the study and contributed to the creation of the original draft of the survey. $\mathrm{KH}$ designed the study, participated in coordination of the study, and helped draft the survey and finalized the manuscript, figures and tables. All authors read and approved the final manuscript.

\section{Acknowledgements}

The authors would like to thank Elliott Kruse for assistance with statistical analysis, Amy Song for assistance with coding data, Dr. Robin DiMatteo for comments on the manuscript and Dr. Jung Park for offsite data collection.

Received: 19 February 2013 Accepted: 12 June 2013

Published: 1 July 2013

\section{References}

1. Kaiser L, Allen LH: Position of the American dietetic association: nutrition and lifestyle for a healthy pregnancy outcome (vol 108, pg 553, 2008). J Am Diet Assoc 2010, 110(1):141-141.

2. Keen $C L$, et al: The plausibility of micronutrient deficiencies being a significant contributing factor to the occurrence of pregnancy complications. J Nutr 2003, 133(5 Suppl 2):1597S-1605S.

3. Streitfeld PP: Congenital-Malformation - teratogenic foods and additives. Birth and the Family Journal 1978, 5(1):7-19.

4. Moore CU, Brodie JL: The relation of maternal to hemorrhage in the new-born. Am J Dis Child 1927, 34(1):53-60.

5. Langley-Evans SC, Welham SJM, Jackson AA: Fetal exposure to a maternal low protein diet impairs nephrogenesis and promotes hypertension in the rat. Life Sci 1999, 64(11):965-974.

6. Gopalakrishnan GS, et al: Influence of maternal pre-pregnancy body composition and diet during early-mid pregnancy on cardiovascular function and nephron number in juvenile sheep. Br J Nutr 2005, 94(6):938-947.

7. Devereux $\mathrm{G}$, et al: Maternal vitamin $\mathrm{D}$ intake during pregnancy and early childhood wheezing. Am J Clin Nutr 2007, 85(3):853-859.

8. Kesby JP, et al: Developmental vitamin D deficiency alters MK 801induced hyperlocomotion in the adult rat: an animal model of schizophrenia. Biol Psychiatry 2006, 60(6):591-596.

9. Calvani $\mathrm{M}$, et al: Consumption of fish, butter and margarine during pregnancy and development of allergic sensitizations in the offspring: role of maternal atopy. Pediatr Allergy Immunol 2006, 17(2):94-102.

10. Hollingsworth JW, et al: In utero supplementation with methyl donors enhances allergic airway disease in mice. J Clin Invest 2008, 118(10):3462-3469.

11. Mcgarvey ST, et al: Maternal prenatal dietary potassium, calcium, magnesium, and infant blood-pressure. Hypertension 1991, 17(2):218-224.

12. Lidow MS: Prenatal cocaine exposure adversely affects development of the primate cerebral-cortex. Synapse 1995, 21(4):332-341. 
13. Chang $L$, et al: Smaller subcortical volumes and cognitive deficits in children with prenatal methamphetamine exposure. Psychiatry ResearchNeuroimaging 2004, 132(2):95-106.

14. Lu R, et al: Effects of prenatal cocaine and heroin exposure on neuronal dendrite morphogenesis and spatial recognition memory in mice. Neurosci Lett 2012, 522(2):128-133.

15. Spohr HL, Willms J, Steinhausen HC: Prenatal alcohol exposure and longterm developmental consequences. Lancet 1993, 341(8850):907-910.

16. Ernst M, Moolchan ET, Robinson ML: Behavioral and neural consequences of prenatal exposure to nicotine. J Am Acad Child Adolesc Psychiatry 2001, 40(6):630-641.

17. El Shawa H, Huffman KJ: Early neocortical development in a FASD mouse model, Program No. 685.09. 2011 Neuroscience Meeting Planner. Washington, DC: Society for Neuroscience, 2011; 2011.

18. Santiago SE, Huffman KJ: Postnatal effects of prenatal nicotine exposure on body weight, brain size and cortical connectivity in mice. Neurosci Res 2012, 73(4):282-291.

19. Aaronson LS, Macnee CL: Tobacco, alcohol, and caffeine use during pregnancy. J Obstet Gynecol Neonatal Nurs 1989, 18(4):279-287.

20. Streissguth AP, Barr HM, Sampson PD: Moderate prenatal alcohol exposure - effects on child lq and learning-problems at age 71/2 years. Alcohol Clin Exp Res 1990, 14(5):662-669.

21. Slotkin TA: Fetal nicotine or cocaine exposure: which one is worse? J Pharmacol Exp Ther 1998, 285(3):931-945.

22. Diekman ST, et al: A survey of obstetrician-gynecologists on their patients' alcohol use during pregnancy. Obstet Gynecol 2000, 95(5):756-763.

23. Payne J, et al: Health professionals' knowledge, practice and opinions about fetal alcohol syndrome and alcohol consumption in pregnancy. Aust N Z J Public Health 2005, 29(6):558-564.

24. Loomans $E M$, et al: Caffeine intake during pregnancy and risk of problem behavior in 5- to 6-year-old children. Pediatrics 2012, 130(2):p. e305-13.

25. Grandjean $P$, et al: Cognitive deficit in 7-year-old children with prenatal exposure to methylmercury. Neurotoxicol Teratol 1997, 19(6):417-428.

26. Ribas-Fito $N$, et al: Polychlorinated biphenyls (PCBs) and neurological development in children: a systematic review. J Epidemio/ Community Health 2001, 55(8):537-546.

27. Vom Saal FS, et al: A physiologically based approach to the study of bisphenol $A$ and other estrogenic chemicals on the size of reproductive organs, daily sperm production, and behavior. Toxicol Ind Health 1998 14(1-2):239-260

28. Braun JM, et al: Prenatal bisphenol A exposure and early childhood behavior. Environ Health Perspect 2009, 117(12):1945-1952.

29. Lamartiniere $C A$, et al: Exposure to the endocrine disruptor bisphenol $A$ alters susceptibility for mammary cancer. Horm Mol Biol Clin Investig 2011, 5(2):45-52.

30. Kristal AR, Shattuck AL, Patterson RE: Differences in fat-related dietary patterns between black, Hispanic and White women: results from the Women's Health Trial Feasibility Study in Minority Populations. Public Health Nutr 1999, 2(3):253-262.

31. Mazur RE, Marquis GS, Jensen HH: Diet and food insufficiency among Hispanic youths: acculturation and socioeconomic factors in the third national health and nutrition examination survey. Am J Clin Nutr 2003, 78(6):1120-1127.

32. Guendelman S, Abrams B: Dietary intake among Mexican-American women: generational differences and a comparison with white nonHispanic women. Am J Public Health 1995, 85(1):20-25.

33. Kumanyika SK: Obesity treatment in minorities. In Handbook of Obesity Treatment. Edited by Wadden T, Stunkard A. New York: Gullford Press; 2002:416-446.

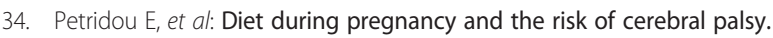
Br J Nutr 1998, 79(5):407-412

35. U.S. Department of Health and Human Services and U.S. Department of Agriculture: Dietary Guidelines for Americans, 2005. 6th edition. Washington, DC: U.S. Government Printing Office; 2005.

36. Yang $M G$, et al: Mammary transfer of $\mathrm{Hg}-203$ from mothers to brains of nursing rats. Proc Soc Exp Biol Med 1973, 142(2):723-726.

37. Choi BH: The effects of methylmercury on the developing brain. Prog Neurobiol 1989, 32(6):447-470.

38. Guzzi G, La Porta CAM: Molecular mechanisms triggered by mercury. Toxicology 2008, 244(1):1-12.
39. Hightower JM, Moore D: Mercury levels in high-end consumers of fish. Environ Health Perspect 2003, 111(4):604-608.

40. Jedrychowski W, et al: Effects of prenatal exposure to mercury on cognitive and psychomotor function in one-year-old infants: epidemiologic cohort study in Poland. Ann Epidemiol 2006, 16(6):439-447.

41. FDA (Food and Drug Administration): Mercury Levels in Commercial Fish and Shellfish (1990-2010); 2010. Available: http://www.fda.gov/Food/ FoodbornelllnessContaminants/Metals/ucm115644.htm [accessed 11 February 2013].

42. Consumer Reports: Mercury in canned tuna still a concern, Consumer Reports magazine; 2011. Available: http://www.consumerreports.org/cro/magazinearchive/2011/january/food/mercury-in-tuna/overview/index.htm [accessed 11 February 2013].

43. Easton MDL, Luszniak D, Von der Geest E: Preliminary examination of contaminant loadings in farmed salmon, wild salmon and commercial salmon feed. Chemosphere 2002, 46(7):1053-1074.

44. Fein GG, et al: Prenatal exposure to polychlorinated-biphenyls - effects on birth size and gestational-age. J Pediatr 1984, 105(2):315-320.

45. Patandin S, et al: Effects of environmental exposure to polychlorinated biphenyls and dioxins on cognitive abilities in Dutch children at 42 months of age. J Pediatr 1999, 134(1):33-41.

46. Jacobson J, Jacobson SW: Specific effects of prenatal PCB exposure on attention and behavior in school age children. Neurotoxicology 2003, 24(2):302-302.

47. Agency for Toxic Substances and Disease Registry (ATSDR): Toxicological profile for Polychlorinated Biphenyls (PCBS). Atlanta, GA: U.S. Department of Health and Human Services, Public Health Service; 2000.

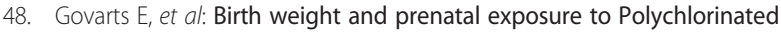
Biphenyls (PCBs) and Dichlorodiphenyldichloroethylene (DDE): a metaanalysis within 12 European birth cohorts. Environ Health Perspectives 2012, 120(2):162-170.

49. Vandenberg LN, et al: Human exposure to bisphenol A (BPA). Reprod Toxicol 2007, 24(2):139-177.

50. Kubwabo C, et al: Migration of bisphenol A from plastic baby bottles, baby bottle liners and reusable polycarbonate drinking bottles. Food Additives and Contaminants Part a-Chemistry Analysis Control Exposure \& Risk Assessment 2009, 26(6):928-937.

51. Brotons JA, et al: Xenoestrogens released from lacquer coatings in food cans. Environ Health Perspect 1995, 103(6):608-612.

52. Yoshida $T$, et al: Determination of bisphenol $A$ in canned vegetables and fruit by high performance liquid chromatography. Food Additives and Contaminants Part a-Chemistry Analysis Control Exposure \& Risk Assessment 2001, 18(1):69-75.

53. Casey BM, et al: Pregnancy outcomes in women with gestational diabetes compared with the general obstetric population - reply. Obstet Gynecol 1998, 91(4):639-640.

54. Major CA, et al: The effects of carbohydrate restriction in patients with diet-controlled gestational diabetes. Obstet Gynecol 1998, 91(4):600-604.

55. Rosenberg TJ, et al: Maternal obesity and diabetes as risk factors for adverse pregnancy outcomes: differences among 4 racial/ethnic groups. Am J Public Health 2005, 95(9):1545-1551.

56. Zhang $\mathrm{CL}$, et al: Dietary fiber intake, dietary glycemic load, and the risk for gestational diabetes mellitus. Diabetes Care 2006, 29(10):2223-2230.

57. Lenders CM: Effect of high-sugar intake by low-income pregnant adolescents on infant birth-weight. J Adolesc Health 1994, 15(7):596-602.

58. Rhoads-Baeza M: Assessing the knowledge, attitudes, and behaviors of pregnant Hispanic women: developing an effective educational intervention for gestational diabetes. ProQuest: Dissertation; 2008.

59. Blank A, Grave GD, Metzger BE: Effects of gestational diabetes on perinatal morbidity reassessed - report of the international workshop on adverse perinatal outcomes of gestational diabetes-mellitus, December 3-4, 1992. Diabetes Care 1995, 18(1):127-129.

60. Lenders $C M$, et al: Gestational age and infant size at birth are associated with dietary sugar intake among pregnant adolescents. J Nutr 1997, 127(6):1113-1117.

61. Carmichael SL, et al: Neural tube defects in offspring associated with maternal periconceptional dietary intake of simple sugars and glycemic index. Am J Epidemiol 2003, 157(11):S33-S33.

62. Tamashiro KLK, et al: Prenatal stress or high-fat diet increases susceptibility to diet-induced obesity in rat offspring. Diabetes 2009, 58(5):1116-1125. 
63. Vucetic $Z$, et al: Maternal high-fat diet alters methylation and gene expression of dopamine and opioid-related genes. Endocrinology 2010, 151(10):4756-4764.

64. Karrdullien $\mathrm{V}$, Bloomquist $\mathrm{E}$ : Influence of prenatal salt on the development of hypertension by Spontaneously Hypertensive Rats (Shr). Proc Soc Exp Biol Med 1979, 160(4):421-425.

65. Porter JP, King SH, Honeycutt AD: Prenatal high-salt diet in the SpragueDawley rat programs blood pressure and heart rate hyperresponsiveness to stress in adult female offspring. Am J Physiol Regul Integr Comp Physiol 2007, 293(1):R334-R342.

66. EWG, Downey - City: Drinking Water Quality Report, Environmental Working Group. Los Angeles, CA: Water Dept; 2011

67. Bove FJ, et al: Public drinking-water contamination and birth outcomes. Am J Epidemiol 1995, 141(9):850-862.

68. Klotz JB, Pyrch LA: Neural tube defects and drinking water disinfection by-products. Epidemiology 1999, 10(4):383-390.

69. Rahman A, et al: Association of arsenic exposure during pregnancy with fetal loss and infant death: a cohort study in Bangladesh. Am J Epidemiol 2007, 165(12):1389-1396.

70. May PA, Gossage JP: Estimating the prevalence of fetal alcohol syndrome. A summary. Alcohol Res Health 2001, 25(3):159-167.

71. Sampson PD, et al: Incidence of fetal alcohol syndrome and prevalence of alcohol-related neurodevelopmental disorder. Teratology 1997 56(5):317-326

72. Steinhausen HC, Willms J, Spohr HL: Correlates of psychopathology and intelligence in children with fetal alcohol syndrome. J Child Psychol Psychiatry 1994, 35(2):323-331.

73. Jacobson SW: Specificity of neurobehavioral outcomes associated with prenatal alcohol exposure. Alcohol Clin Exp Res 1998, 22(2):313-320.

74. Mattson SN, Riley EP: A review of the neurobehavioral deficits in children with fetal alcohol syndrome or prenatal exposure to alcohol. Alcohol Clin Exp Res 1998, 22(2):279-294.

75. Cone-Wesson B: Prenatal alcohol and cocaine exposure: influences on cognition, speech, language, and hearing. J Commun Disord 2005, 38(4):279-302.

76. Riley EP, Infante MA, Warren KR: Fetal alcohol spectrum disorders: an overview. Neuropsychol Rev 2011, 21(2):73-80.

77. CDC: Alcohol usage and binge drinking among women of childbearing age. In Morbidity and Mortality Weekly Report. 2012, Centers for Disease Control and Prevention. 2012:534-538.

78. Zeisel SH: Importance of methyl donors during reproduction. Am J Clin Nutr 2009, 89(2):673S-6775.

79. Buhusi CV, Lamoureux JA, Meck WH: Prenatal choline supplementation increases sensitivity to contextual processing of temporal information. Brain Res 2008, 1237:204-212.

80. Smithells RW, Sheppard S, Schorah CJ: Vitamin deficiencies and neural tube defects. Arch Dis Child 1976, 51(12):944-950.

81. Schmidt RJ, et al: Prenatal vitamins, one-carbon metabolism gene variants, and risk for autism. Epidemiology 2011, 22(4):476-485.

82. Fujii $T$, Sasaki H, Nishimura H: Teratogenicity of caffeine in mice related to its mode of administration. Jpn J Pharmacol 1969, 19(1):134-138.

83. Borlee I, et al: Coffee, risk factor during pregnancy. Louvain Medical 1978, 97(5):279-284

84. Goyan J: Food and Drug Administration news release no P80-36. Washington DC: FDA; 1980

85. American Pregnancy Association: Caffeine intake during pregnancy; 2013. http://americanpregnancy.org/pregnancyhealth/caffeine.html.

86. Martin JC: Maternal nicotine and caffeine consumption and offspring outcome - an overview. Neurobehav Toxicol Teratol 1982, 4(4):421-427.

87. Sobotka TJ: Neuro-behavioral effects of prenatal caffeine. Ann N Y Acad Sci 1989, 562:327-339.

88. Bekkhus $M$, et al: Intrauterine exposure to caffeine and inattention/ overactivity in children. Acta Paediatr 2010, 99(6):925-928.

89. Leviton $A$, Cowan $L$ : A review of the literature relating caffeine consumption by women to their risk of reproductive hazards. Food Chem Toxicol 2002, 40(9):1271-1310.

90. Wisborg K, et al: Maternal consumption of coffee during pregnancy and stillbirth and infant death in first year of life: prospective study. BMJ 2003, 326(7386):420.

91. Bech $\mathrm{BH}$, et al: Coffee and fetal death: a cohort study with prospective data. Am J Epidemiol 2005, 162(10):983-990.
92. Mitchell AA: Medication use during pregnancy, with particular focus on prescription drugs: 1976-2008. Am J Obstet Gynecol 2011, 205(51):e1-8.

93. Trasler DG: Aspirin-induced cleft lip and other malformations in mice. Lancet 1965, 1(7385):606.

94. Burdan F, et al: Congenital ventricular septal defects and prenatal exposure to cyclooxygenase inhibitors. Braz J Med Biol Res 2006, 39(7):925-934.

95. Streissguth $\mathrm{AP}$, et al: Aspirin and acetaminophen use by pregnant-women and subsequent child IQ and attention decrements. Teratology 1987, 35(2):211-219.

96. Broussard CS, Rasmussen SA, Friedman JM: Maternal treatment with opioid analgesics and risk for birth defects: additional considerations. Am J Obstet Gynecol 2011, 205(3):E12-E13.

97. Bracken MB: Drug-use in pregnancy and congenital heart-disease in offspring. N Eng J Med 1986, 314(17):1120-1120.

98. CDC: Trends in infant mortality attributable to birth defects-United States, 1980-1995. In Morbidity and Mortality Weekly Report; 1998:773-778,

99. Robison LL, et al: Maternal drug-use and risk of childhood nonlymphoblastic leukemia among offspring an epidemiologic investigation implicating marijuana. Cancer 1989, 63(10):1904-1911.

doi:10.1186/1475-2891-12-91

Cite this article as: Santiago et al:: Consumption habits of pregnant women and implications for developmental biology: a survey of predominantly Hispanic women in California. Nutrition Journal 2013 12:91.

\section{Submit your next manuscript to BioMed Central and take full advantage of:}

- Convenient online submission

- Thorough peer review

- No space constraints or color figure charges

- Immediate publication on acceptance

- Inclusion in PubMed, CAS, Scopus and Google Scholar

- Research which is freely available for redistribution 